\title{
BMJ Open Meta-analysis of anti-Saccharomyces cerevisiae antibodies as diagnostic markers of Behçet's disease with gastrointestinal involvement
}

\author{
Linlin Cheng (1) , Liubing Li, Chenxi Liu, Songxin Yan, Yongzhe Li (1)
}

To cite: Cheng L, Li L, Liu C, et al. Meta-analysis of antiSaccharomyces cerevisiae antibodies as diagnostic markers of Behçet's disease with gastrointestinal involvement. BMJ Open 2020;10:e033880. doi:10.1136/ bmjopen-2019-033880

\section{- Prepublication history and} additional material for this paper are available online. To view these files, please visit the journal online (http://dx.doi. org/10.1136/bmjopen-2019033880).

Received 26 August 2019 Revised 15 May 2020 Accepted 09 July 2020
Check for updates

(c) Author(s) (or their employer(s)) 2020. Re-use permitted under CC BY-NC. No commercial re-use. See rights and permissions. Published by BMJ.

Department of Clinical Laboratory, Peking Union Medical College Hospital, Peking Union Medical College and Chinese Academy of Medical Sciences, Beijing, China

Correspondence to Dr Yongzhe Li; yongzhelipumch@126.com

\section{ABSTRACT}

Objective Due to common exposure to yeast in the alcoholic and baking industry, positive rate of anti-Saccharomyces cerevisiae antibodies (ASCA) is reportedly high in patients with Behçet's disease (BD) who have gastrointestinal symptoms (gastrointestinal $\mathrm{BD}(\mathrm{GIBD}))$. We performed a meta-analysis to assess the diagnostic value of ASCA in differentiating patients with BD from those with other chronic inflammatory bowel diseases.

Methods The meta-analysis is presented with the Preferred Reporting Items for Systematic Reviews and Meta-Analyses and Meta-analysis of Observational Studies in Epidemiology checklist. Relevant studies that investigated ASCA levels in patients with BD were retrieved from PubMed, EMBASE, Web of Science, SCOPUS and the Cochrane Library on 12 July 2019; the search was rerun on 12 February 2020. Stata/SE V.12.0 and Meta-DiSc V.1.4 were used to perform the meta-analysis and sensitivity analysis, disaggregated by isotypes of ASCA.

Results Nine studies were included in the metaanalysis. The results revealed a strong association between ASCA and GIBD, especially ASCA-IgG $(0 R=5.50$ (95\% Cl 2.58 to 11.55), $p=0.000$ ) and $A S C A-\lg G+\lg A$ (OR=5.36 (95\% Cl 1.40 to 20.45), $p=0.014)$. The positivity rate of ASCA in GIBD was significantly higher than that in ulcerative colitis (UC): $\lg A(O R=2.13(95 \%$ $\mathrm{Cl} 1.30$ to 3.50$), \mathrm{p}=0.003) ; \lg \mathrm{G}+\lg \mathrm{A}(\mathrm{OR}=2.19(95 \% \mathrm{Cl}$ 1.03 to 4.66), $p=0.042) ; \lg \mathrm{G} / \operatorname{lgA}((=2.03$ (95\% Cl 1.30 to 3.17), $p=0.002)$. However, the frequency of ASCA-IgG was significantly higher in patients with Crohn's disease than GIBD (OR=0.48 (95\% $\mathrm{Cl} 0.28$ to 0.83$), \mathrm{p}=0.009)$. There was no significant difference in ASCA positivity between $\mathrm{BD}$ without gastrointestinal involvement and healthy controls and between GIBD and intestinal tuberculosis (iTB) $(p>0.05)$.

Conclusion ASCA may play a role in the pathogenesis of gastrointestinal involvement. Negative result of $\mathrm{lgG}$ favours the diagnosis of GIBD/BD when differentiated from Crohn's disease. ASCA-IgA showed moderate diagnostic performance in distinguishing GIBD and UC and the diagnostic performance was better in combination with IgG. However, ASCA may not be a useful serologic marker distinguishing GIBD and iTB.

PROSPERO registration number CRD42020115245.
Strengths and limitations of this study

- In addition to the healthy controls, we included patients with other gastrointestinal diseases that are considered in the differential diagnosis of gastrointestinal Behçet's disease (BD) in clinical settings (such as ulcerative colitis, Crohn's disease, and intestinal tuberculosis), in order to improve the clinical awareness of anti-Saccharomyces cerevisiae antibodies (ASCA).

- Inclusion of both categorical data (positivity rate) and continuous data (serum concentration) pertaining to ASCA increases the reliability of the results of meta-analysis.

- We separately performed meta-analysis of $\lg G, \lg A$ and $\lg \mathrm{G}+\lg A$, which provides insights into their ability to differentiate $\mathrm{BD}$ from other gastrointestinal diseases.

- Comprehensive summary of evidence linking ASCA and autoimmune diseases provides preliminary insights into the pathogenicity of $S$. cerevisiae.

- Analysis of too many subgroups contributed to potential heterogeneity due to the small number of studies included in each subgroup.

\section{INTRODUCTION}

Behçet's disease (BD) is a chronic systemic vascular autoimmune/inflammatory disease with a high propensity for recurrence; the pathogenetic mechanisms of this disease are not well elucidated. ${ }^{1}$ Virtually no specific histological or laboratory features of BD have been identified. Therefore, the diagnosis of BD is typically challenging as it is mainly based on clinical features. ${ }^{2} 3$ The diagnosis is frequently delayed until the development of clinical manifestations that qualify the diagnostic criteria. The estimated duration between the onset of symptoms and the fulfilment of diagnostic criteria is approximately 4 years. $^{4}$

Moreover, patients with prominent involvement of a particular organ system are easily misdiagnosed. For example, patients who 
have gastrointestinal symptoms as the main manifestation are liable to be misdiagnosed as having Crohn's disease (CD), ulcerative colitis (UC) or intestinal tuberculosis (iTB). These features make formulating disease criteria difficult, causing deleterious effects on the patients.

Several recent studies (but not all) have reported the diagnostic value of anti-Saccharomyces cerevisiae antibodies (ASCA) in BD. S. cerevisiae, also known as the baker's or brewer's yeast, has long been used to ferment the sugars in cereals to produce alcoholic beverages; it is also used in the baking industry to raise dough. As a consequence, we are now commonly exposed to yeast. ${ }^{5}$ IgG and IgA antibodies against the phosphopeptidomannan of the $S$. cerevisiae cell wall have been discovered as autoantibodies in the sera of patients with $\mathrm{BD}$, especially those with gastrointestinal involvement. This suggests a role of environmental stimuli in the pathogenesis of $\mathrm{BD}$. However, patients with inflammatory bowel disease such as $\mathrm{CD}$ also have a high prevalence rate of ASCA due to their similarities. ${ }^{6-11}$ In this context, identification of ASCA as a diagnostic marker for BD is a key imperative. The objectives of this study were to summarise the findings pertaining to the relevance of ASCA in $\mathrm{BD}$ and other gastrointestinal diseases and to perform a meta-analysis to assess its diagnostic accuracy for BD.

\section{METHODS}

\section{Study design}

The Preferred Reporting Items for Systematic Reviews and Meta-Analysis Diagnostic Test Accuracy guidelines ${ }^{12}$ (online supplemental file 1) and Meta-analysis of Observational Studies in Epidemiology ${ }^{13}$ (online supplemental file 2) were followed throughout the literature search process to structure and design the framework for the review. $^{14}$

\section{Literature search}

A comprehensive literature search was performed to identify studies pertaining to ASCA as biomarkers for BD in five biomedical databases, that is, PubMed, EMBASE, Web of Science, SCOPUS and the Cochrane Library on July 12, 2019. The search terms for Behçet's disease were: Behcet, triple symptom complex, triple symptom complices, Adamantiades Behcet and old silk route disease; the search terms for Saccharomyces cerevisiae were: S. cerevisiae, Saccaromyces cerevisiae, Saccharomyces capensis, Saccharomyces diastaticus, Saccharomyces italicus, Saccharomyces oviformis, Saccharomyces uvarum, brewer yeast or baker yeast, mannan, manna, polymannan, glucomannan, yeast mannan, dicoman, humamil, ASCA. Combination of keywords using 'AND' was used to retrieve studies in the range of 'all fields' or 'all text'. The search was rerun on 12 February 2020 to ensure inclusion of recent studies. No restrictions were imposed with respect to time of publication, region or ethnicity of the study population. In addition, the reference list of obtained articles was also examined to identify possible relevant studies. The full search strategy for EMBASE is shown in online supplemental file 3 .

\section{Eligibility and exclusion criteria}

The inclusion criteria were: (1) studies that evaluated the diagnostic accuracy of ASCA in BD; (2) availability of adequate data pertaining to the prevalence rate or serum levels of ASCA in patients with BD; (3) studies with healthy population and/or disease controls and (4) meeting abstracts or letters to the editor were also included.

The exclusion criteria were: (1) studies with incomplete data; (2) review articles; (3) non-English articles and (4) in case of studies with overlapping study population, studies with smaller sample size were excluded. Two investigators independently performed the literature search, screened the titles and abstracts, followed by fulltext review of eligible studies.

\section{Data extraction and quality assessment}

Two independent investigators reviewed the full-text articles, extracted the data and assessed the study quality using the Quality Assessment of Diagnostic Accuracy Studies (QUADAS-2). The included items were evaluated as yes, no or uncertain. Inter-researcher disagreements were resolved by consensus, or by a third investigator. Data pertaining to the following variables were extracted: publication year, article type, first author's name, country, isotypes of ASCA detected, age and sex, research design, sample size, experimental method, trade names of experimental materials, cut-off values, diagnostic criteria and serum titres and/or prevalence rate of ASCA in BD, gastrointestinal BD (GIBD), healthy controls (HC), patients with $\mathrm{CD}, \mathrm{UC}$ and iTB. The data were either obtained directly from the article, calculated or requested from the author via email.

\section{Statistical analysis}

Pooled OR with $95 \%$ CIs were calculated to evaluate the association between ASCA and BD (without gastrointestinal involvement)/GIBD/CD/UC/iTB using Stata/SE V.12.0. Meta-DiSc V.1.4 was used to calculate the sensitivity and specificity to assess the overall diagnostic performance of ASCA. Heterogeneity among the included studies was evaluated using the Cochran's Q-statistic. P values $>0.10$ were considered indicative of lack of significant heterogeneity. We chose the random effects models (REM) since REM tends to generalise findings beyond the included studies by assuming that the selected studies are random samples from a larger population. ${ }^{15}$ Subgroup analysis was performed disaggregated by the isotypes of ASCA and different disease controls. The isotypes of ASCA were classified and defined as follows: IgG, IgA, IgG/IgA (positive results of either IgG or IgA) and IgG+IgA (positive results of both IgG and IgA).

In order to increase the robustness of the meta-analysis, we also extracted the data pertaining to serum levels of ASCA from five studies and performed meta-analysis using the Continuous data module of Stata/SE V.12.0. 
The REM was used for the analysis and weighted mean difference (WMD) was used as the effect measure if the same unit was used in these studies and there were minor differences with respect to the serum levels of ASCA. Sensitivity analysis was performed using Stata/SE V.12.0 to evaluate the stability of the results after sequential exclusion of one study at a time.

\section{Patient and public involvement}

The present study was a meta-analysis and systematic review based on published data. Patients and public were not involved in the study design, conduct, data analysis and result dissemination.

\section{Relationship between ASCA and autoimmune disease}

We searched PubMed for studies pertaining to the relationship between ASCA and autoimmune diseases. The two search terms used were autoimmune disease and $S$. cerevisiae. We performed an interval statistic of four indicators of ASCA—sensitivity, specificity, positive likelihood (LR+) and negative likelihood (LR-) based on the included studies sorted by diseases.

\section{RESULTS}

\section{Literature search and characteristics of studies}

A total of 625 documents were retrieved on database and manual search. Fifty-one duplicate publications were excluded using the document management software. A total of 127 records were retained after screening of titles and/or abstracts; the excluded records included review articles, animal model studies, therapeutic or drug research, genetic research, book chapters, duplicate publications not recognised by software and other irrelevant records. After full-text review for eligibility, 22 records were selected. Finally, we included nine available studies with adequate data in the meta-analysis (figure 1 ). Two studies were included after obtaining the relevant data by contacting the respective authors. ${ }^{9}{ }^{10}$ In addition, we also verified two studies ${ }^{16} 17$ with overlapping study population; of these, only one study was included in the meta-analysis. Three studies ${ }^{6818}$ were presented as meeting abstracts without adequate data to allow the construction of a $2 \times 2$ table. One article $^{7}$ was a letter to the editor and only reported the prevalence rate of ASCA antibody in patients with $\mathrm{BD}$, without information about the control group. One study ${ }^{19}$ had employed a unique calculation method and could not be included in the meta-analysis. Among the included studies, there were 200 cases of BD, 288 cases of GIBD, 471 cases of CD, 552 cases of UC, 112 cases of iTB and 391 HCs (table 1 and online supplemental file 4 ).

\section{Quality assessment}

There were eight case-control studies and one retrospective study. ${ }^{9}$ The results of quality assessment by QUADAS-2 including the risk of bias and applicability concerns pertaining to each domain ${ }^{20}$ are shown in online supplemental file 5. The results indicated that the included

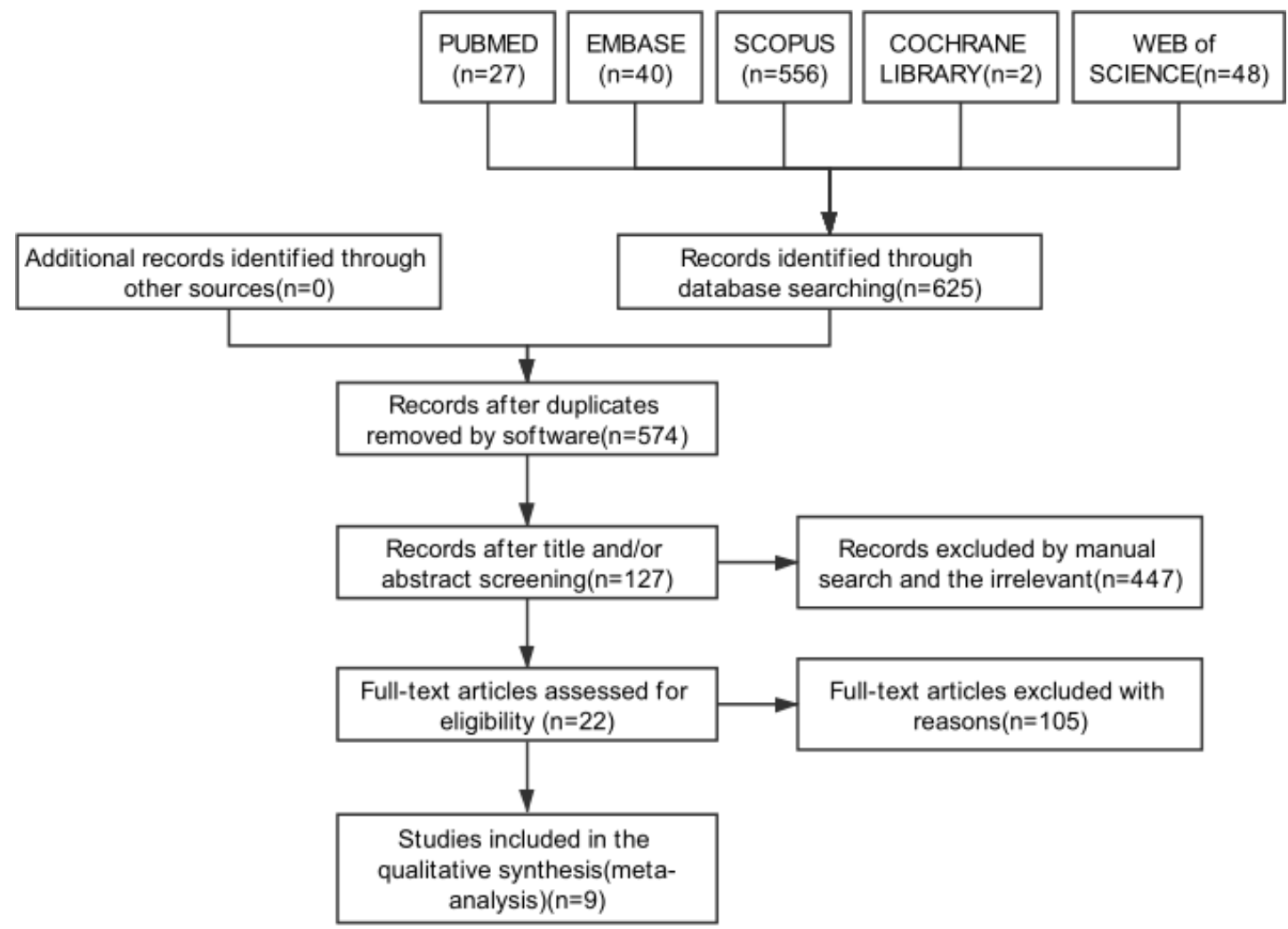

Figure 1 Preferred Reporting Items for Systematic Reviews and Meta-Analysis flow diagram illustrating the literature screening process and the criteria for inclusion of studies in the meta-analysis. 


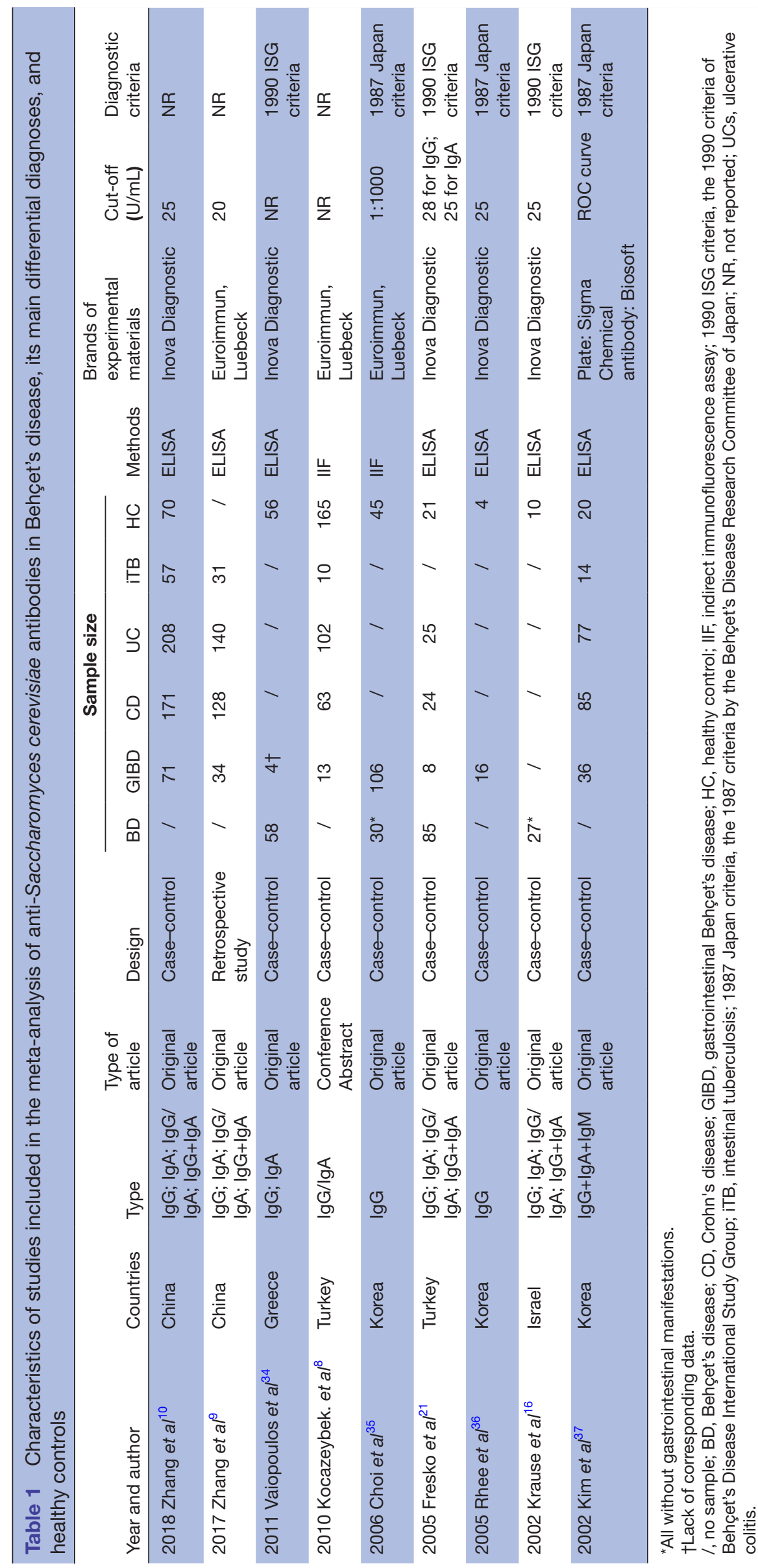


Table 2 Association between the presence of ASCA and BD (without gastrointestinal symptom)/GIBD and other intestinal diseases

\begin{tabular}{|c|c|c|c|c|}
\hline Subgroup & Antibody & Number of studies & $\begin{array}{l}\text { Diagnostic OR } \\
(95 \% \mathrm{Cl})\end{array}$ & Significance test (p) \\
\hline \multirow[t]{4}{*}{$\mathrm{BD}$ vs HC } & ASCA-IgG & 4 & 1.00 (0.28 to 3.53$)$ & 0.997 \\
\hline & ASCA-IgA & 2 & 2.50 (0.63 to 9.96$)$ & 0.194 \\
\hline & ASCA-lgG+lgA & 2 & 1.06 (0.17 to 6.78$)$ & 0.954 \\
\hline & ASCA-lgG/lgA & 2 & 2.88 (0.62 to 13.44$)$ & 0.179 \\
\hline \multirow[t]{4}{*}{ GIBD vs HC } & ASCA-IgG & 3 & 5.50 (2.58 to 11.55$)$ & 0.000 \\
\hline & ASCA-IgA & 2 & 2.65 (1.18 to 5.96$)$ & 0.018 \\
\hline & ASCA-lg $G+\lg A$ & 2 & 5.36 (1.40 to 20.45$)$ & 0.014 \\
\hline & ASCA-lgG/lgA & 3 & 2.90 (1.47 to 5.74$)$ & 0.002 \\
\hline \multirow[t]{4}{*}{ GIBD vs CD } & ASCA-IgG & 3 & 0.48 (0.28 to 0.83$)$ & 0.009 \\
\hline & ASCA-IgA & 3 & 0.91 (0.56 to 1.46$)$ & 0.685 \\
\hline & ASCA- $\lg G+\lg A$ & 3 & 0.58 (0.30 to 1.11$)$ & 0.100 \\
\hline & ASCA-lgG/lgA & 4 & 0.57 (0.28 to 1.15$)$ & 0.117 \\
\hline \multirow[t]{4}{*}{ GIBD vs UC } & ASCA-IgG & 3 & 1.78 (0.98 to 3.22$)$ & 0.057 \\
\hline & ASCA-IgA & 3 & 2.13 (1.30 to 3.50$)$ & 0.003 \\
\hline & ASCA-lgG+lgA & 3 & 2.19 (1.03 to 4.66$)$ & 0.042 \\
\hline & ASCA-lgG/lgA & 4 & 2.03 (1.30 to 3.17$)$ & 0.002 \\
\hline \multirow[t]{4}{*}{ GIBD vs iTB } & ASCA-IgG & 2 & 1.08 (0.50 to 2.32$)$ & 0.854 \\
\hline & ASCA-IgA & 2 & 1.51 (0.71 to 3.22$)$ & 0.290 \\
\hline & ASCA- $\lg G+\lg A$ & 2 & 1.02 (0.40 to 2.62$)$ & 0.972 \\
\hline & ASCA-lgG/lgA & 3 & 1.05 (0.58 to 1.87$)$ & 0.883 \\
\hline
\end{tabular}

ASCA, anti-Saccharomyces cerevisiae antibodies; BD, Behçet's disease without gastrointestinal symptom; CD, Crohn's disease; GIBD, gastrointestinal Behçet's disease; HC, healthy control; iTB, intestinal tuberculosis; UC, ulcerative colitis.

studies were of high quality in general. Overall, none of the nine included studies showed any major methodological bias or flaws, which indicates the robustness of our meta-analysis.

\section{Meta-analysis}

Association between ASCA and BD (without gastrointestinal involvement), GIBD and other intestinal diseases

Data pertaining to correlation between ASCA and BD (without gastrointestinal involvement) $/ \mathrm{GIBD} / \mathrm{CD} / \mathrm{UC} /$ iTB are listed in table 2. No substantial heterogeneity ( $p>0.1$ for all) was observed by using REM to calculate the OR. The results revealed a strong association between all detection types of ASCA and GIBD, especially for ASCA-IgG (OR=5.50 (95\% CI 2.58 to 11.55 ), $\mathrm{p}=0.000$ ) and ASCA-IgG+IgA (OR=5.36 (95\% CI 1.40 to 20.45), $\mathrm{p}=0.014$ ). When comparing GIBD and UC, of the positivity rate for ASCA in GIBD was significantly higher than that for UC: $\operatorname{IgA}(\mathrm{OR}=2.13$ (95\% CI 1.30 to 3.50), $\mathrm{p}=0.003), \operatorname{IgG}+\operatorname{IgA}(\mathrm{OR}=2.19 \quad(95 \%$ CI 1.03 to 4.66$)$, $\mathrm{p}=0.042)$ and $\operatorname{IgG} / \operatorname{IgA}(\mathrm{OR}=2.03$ (95\% CI 1.30 to 3.17$)$, $\mathrm{p}=0.002$ ). Conversely, the frequency of only ASCA-IgG in patients with $\mathrm{CD}$ was significantly higher than that in the GIBD (OR=0.48 (95\% CI 0.28 to 0.83 ), $\mathrm{p}=0.009$ ). Further, on stratified analysis according to detection method,
ASCA-IgG was associated with GIBD using both the ELISA method (OR=3.83 (95\% CI 1.37 to 10.70$), \mathrm{p}=0.010)$ and the indirect immunofluorescence assay (IIF) $(\mathrm{OR}=8.17$ (95\% CI 2.73 to 24.43), $\mathrm{p}=0.000$ ) (figure 2). However, no significant difference was observed with respect to ASCA positivity between BD without gastrointestinal involvement and HC and between GIBD and iTB $(p>0.05)$.

Diagnostic ability of ASCA for GIBD

The overall sensitivity for ASCA-IgG in patients with GIBD detected by IIF was 0.44 , which is much higher than that of ELISA ( 0.20 (95\% CI 0.12 to 0.31$)$ ) (table 3$)$. Combined detection of IgG and IgA by ELISA increased the sensitivity to 0.33 ( $95 \%$ CI 0.23 to 0.44 ). However, we observed a low level of sensitivity of ASCA-IgG/IgA by IIF, which may be attributable to the inclusion of only one study with few patients with GIBD (n=13).

Difference in serum levels of ASCA in GIBD and other intestinal diseases

Serum levels of ASCA-IgA observed in GIBD were significantly greater than that in $\mathrm{HC}(\mathrm{WMD}=7.02 \quad(95 \%$ CI 2.23 to 11.81$), \mathrm{p}=0.004)$ and $\mathrm{UC}(\mathrm{WMD}=5.28(95 \%$ CI 0.39 to 10.17), $\mathrm{p}=0.034)$ in contrast to ASCA-IgG $(\mathrm{p}>0.05)$ (figure 3$)$. On the contrary, serum levels of 


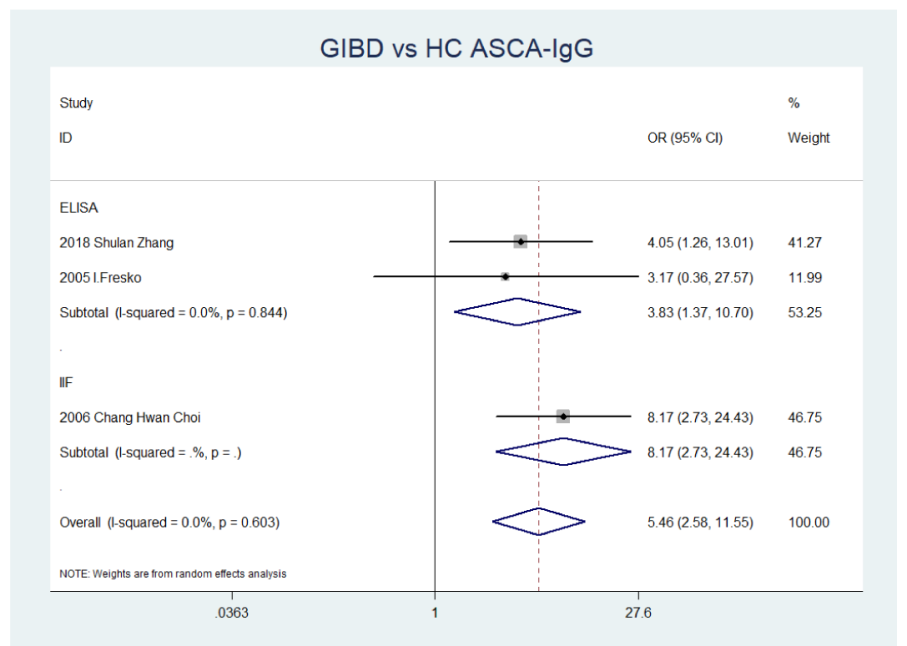

Figure 2 Forest plot of the association between the presence of ASCA-IgG and GIBD stratified by detection methods. ASCA, anti-Saccharomyces cerevisiae antibodies; GIBD, gastrointestinal Behçet's disease; HC, healthy control; IIF, indirect immunofluorescence assay.

ASCA-IgG in CD were significantly greater than that in GIBD (WMD=-11.04 (95\% CI -16.74 to -5.34$), \mathrm{p}=0.000)$ (figure 3). However, we found no significant difference in serum levels of ASCA between BD without gastrointestinal symptoms and HC ( $>0.05)$ (figure 3$)$.

\section{Heterogeneity and sensitivity analysis}

We performed sensitivity analysis to assess the stability of the results. The results showed that the studies by Krause et $a l,{ }^{16}$ Zhang et $a l^{10}{ }^{10}$ Kocazeybe $e t a l^{8}$ and Fresko et $a l^{21}$ were the key contributors to the heterogeneity (online supplemental file 6). Thus, the results of related subgroup analysis are considered to be less stable.

\section{Summary of the relationship of ASCA with autoimmune disease}

Sixteen studies reporting the relevance of ASCA and autoimmune diseases were included in the summary. The sensitivity, specificity, LR+, and LR- of ASCA for different autoimmune diseases are summarised in table 4. Although the diagnostic results of ASCA reported by different studies vary, the summary revealed an overall association between ASCA and autoimmune diseases especially in patients with scleroderma, juvenile idiopathic arthritis, $\mathrm{CD}$ and systemic lupus erythematosus with high sensitivity $(>40 \%)$, high specificity $(>95)$ and high $\mathrm{LR}+(>5)$ (table 4).

\section{DISCUSSION}

\section{Serological markers in BD}

The diagnosis of $\mathrm{BD}$ is typically challenging prior to the appearance of clinical symptoms necessary to qualify the diagnostic criteria. Currently, there are no specific laboratory biomarkers of $\mathrm{BD}$; however, some specific autoantibodies in the context of BD have been reported. Therefore, identification of non-invasive specific diagnostic and prognostic biomarkers of $\mathrm{BD}$ is of much clinical relevance and a key focus area of research.

\section{ASCA in BD and autoimmune diseases}

Several recent studies have investigated the relationship of ASCA with BD or other autoimmune diseases. S. cerevisiae has long been used in alcoholic and baking industry and for the production of vaccines owing to its antigenic component. However, during long-term and ubiquitous presence, even the commensal and classically non-pathogenic microbiota can trigger autoimmunity due to loss of immune tolerance towards the resident bacterial flora, like in gastrointestinal tract. ${ }^{22} 23$ The reported similarity of sequences involving the eukaryotic microorganism and self-antigens suggest a mechanism of molecular mimicry and also the plausibility of shared epitopes in different autoimmune diseases. The production

Table 3 Pooled sensitivity and specificity of ASCA-IgG and IgG/IgA for diagnosis of GIBD assessed by ELISA and IIF

\begin{tabular}{|c|c|c|c|c|}
\hline \multirow{2}{*}{$\begin{array}{l}\text { Methods } \\
\text { Diagnostic accuracy }\end{array}$} & \multicolumn{2}{|c|}{ ELISA } & \multicolumn{2}{|c|}{ IIF } \\
\hline & Pooled sensitivity $(95 \% \mathrm{Cl})$ & Pooled specificity $(95 \% \mathrm{Cl})$ & Sensitivity & Specificity \\
\hline ASCA-IgG & $0.20(0.12$ to 0.31$)$ & 0.93 (0.86 to 0.98$)$ & 0.44 & 0.91 \\
\hline
\end{tabular}

ASCA, anti-Saccharomyces cerevisiae antibodies; GIBD, gastrointestinal Behçet's disease; IIF, indirect immunofluorescence assay. 


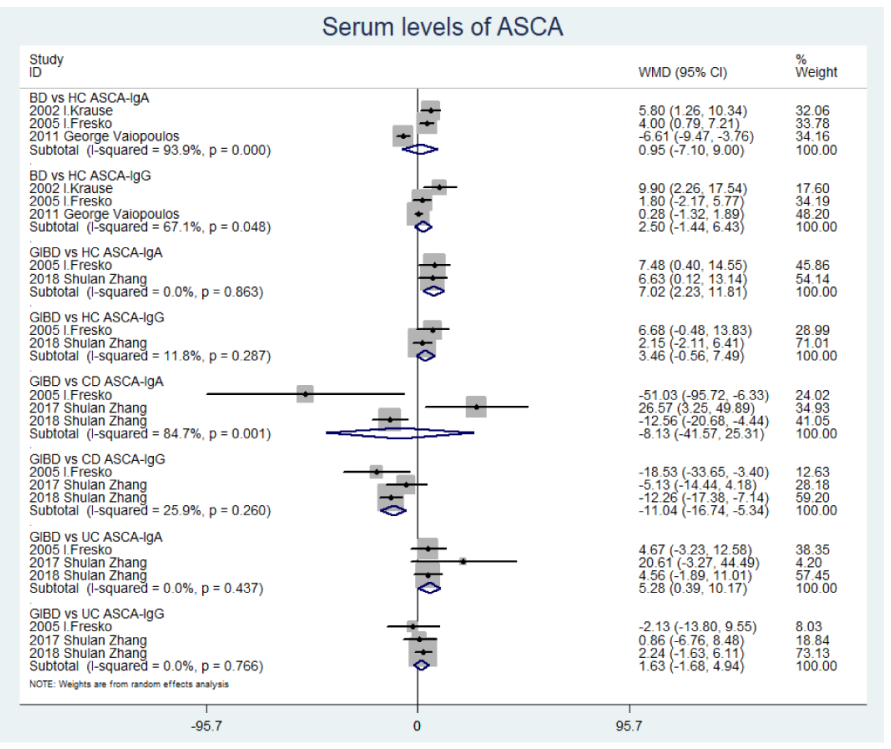

Figure 3 Forest plot comparing serum levels of ASCA between BD without gastrointestinal symptom/GIBD and HC/CD/UC. ASCA, anti-Saccharomyces cerevisiae antibodies; CD, Crohn's disease; GIBD, gastrointestinal Behçet's disease; HC, healthy control; IIF, indirect immunofluorescence assay; UC, ulcerative colitis.

of ASCA by the subsequent activation of the humoral immune response may lead to a direct pathogenic role through a costimulatory CD80/86-CD28mediated effect. ${ }^{22}$ Moreover, healthy family members but not spouses of patients with BD were also found to have increased levels of ASCA, which indicated a role of genetic factors in addition to environmental stimuli. ${ }^{1723}$ A large number of studies have assessed the role of ASCA in the context of several systemic and organ-specific autoimmune diseases, such as BD, scleroderma, systemic lupus erythematosus, primary Sjögren's syndrome, and rheumatoid arthritis (table 4). The results suggest that the relation of ASCA with $\mathrm{BD}$ or other autoimmune diseases may represent a potential pathogenic mechanism between ASCA and autoimmunity; this underlines the importance of ASCA as a valuable serologic marker for autoimmune diseases including BD.

\section{Results of the meta-analysis}

To the best of our knowledge, this is the third metaanalysis of evidence pertaining to autoantibodies in patients with BD after anticardiolipin antibodies. ${ }^{24} 25$ ASCA have been widely researched in BD, CD and other autoimmune disease; in order to investigate the diagnostic value and possible pathogenetic role of ASCA in BD, we included nine studies in this meta-analysis. Among these, some studies included patients with BD with systemic involvement including or excluding gastrointestinal involvement, while others included only patients with BD with gastrointestinal involvement. Therefore, in order to reduce the impact of differences with respect to frequency distribution of gastrointestinal symptoms in each study, we disaggregated patients with BD into those with gastrointestinal involvement only and those without gastrointestinal manifestations. The isotype antibodies of ASCA tested and the results presented by the studies are also different. The meta-analysis revealed a strong association of ASCA with GIBD and not with $\mathrm{BD}$ with no gastrointestinal involvement; this suggests the role of ASCA in the pathogenesis of gastrointestinal involvement. ASCA showed a moderate diagnostic performance as a biomarker for the differential diagnosis between GIBD and CD, and the negative result of ASCA-IgG may slightly favour the diagnosis of GIBD/BD when compared with $\mathrm{CD}$, especially with concomitant positive HLA-B51 tests. ${ }^{26}$ In addition, ASCA-IgA showed a moderate diagnostic value for distinguishing GIBD and UC and would perform better with concomitant detection of IgG. However, ASCA failed to distinguish between GIBD and iTB. Besides, the concomitant evaluation of both continuous data (sensitivity and specificity) and discontinuous data (serum levels) helped increase the credibility of our results.

\section{Heterogeneity}

Combined with the results of QUADAS-2, we found that the heterogeneity in this meta-analysis was largely attributable to the following reasons (see table 1, online supplemental file 5). (1) The different diagnostic criteria used in the included studies. Different criteria may have different thresholds for diagnosis or place more weight on some symptoms than others. Specifically, the 1990 ISG criteria require the presence of oral ulceration plus any two of the following: genital ulceration, typical eye lesions, typical skin lesions, or positive pathergy test for diagnosis of BD. ${ }^{2}$ In contrast, the 1987 Japan criteria require all four characteristics for the diagnosis of $\mathrm{BD}$, that is, oral ulceration, typical eye 


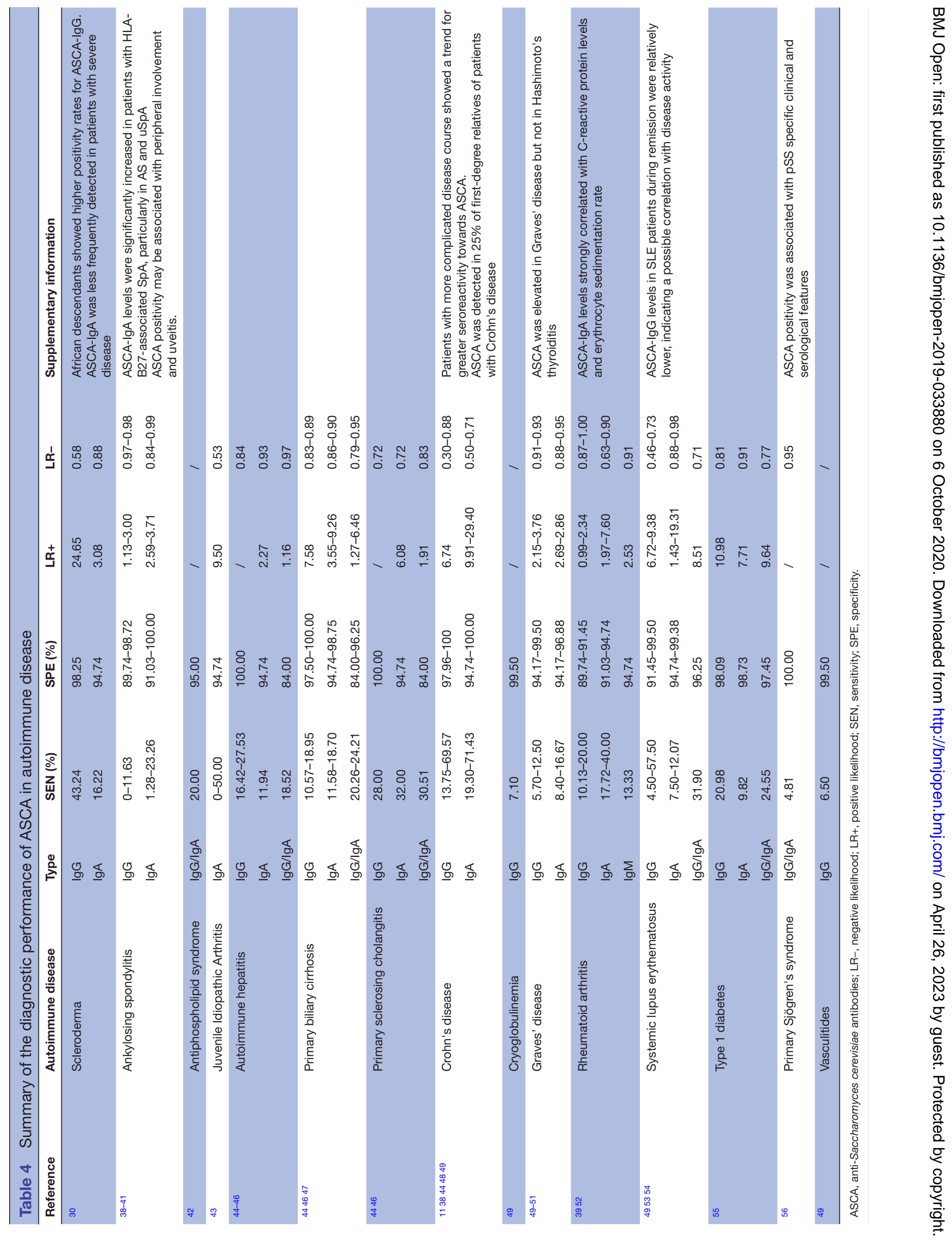


lesions, typical skin lesions, and genital ulceration. ${ }^{27}$ The ISG criteria and the Japanese criteria often fail to classify some patients with $\mathrm{BD}$; in addition, the Japanese criteria may also cause misclassified diagnosis. This may have caused the different diagnostic sensitivity and specificity for $\mathrm{BD}^{28}$ (2) Differences in demographic characteristics of included studies. The clinical features and laboratory findings tend to exhibit wide variability in different populations and clinical settings. ${ }^{29} 30$ However, we failed to perform subgroup analysis disaggregated by ethnicity owing to the small sample size in each subgroup. (3) Different antibody assays and cut-off values. Commercial kits and in-house tests from different laboratories have variable performance, which may affect the diagnosis and management of patients. We found that different methods and cut-off values were adopted by the studies included in this meta-analysis. Notably, there was a significant association between ASCA-IgG and GIBD using both methods; however, it seems that IIF has a higher sensitivity than ELISA. Nonetheless, further investigations with larger study population are required to provide more definitive evidence. Although previous studies have shown that IIF has a better performance, ELISA provides the titre change of serum antibodies and could have an equal performance to IIF by changing cut-off values to optimise the overall diagnostic performance. ${ }^{31}$ (4) According to the QUADAS-2, there are certain concerns that most studies have risk of bias (internal validity) in patient selection, which, to some extent, would cause the distorted estimation in diagnostic accuracy. ${ }^{20}$

\section{Limitations of the meta-analysis}

In order to fully evaluate the value of ASCA for differential diagnosis of $\mathrm{BD}$, we included patients with $\mathrm{CD}, \mathrm{UC}$, and $\mathrm{iTB}$ as the comparison objects in our meta-analysis. However, there are some limitations of this meta-analysis. (1) Grey literature database, paper database, and other language databases were not used for the literature search. Quite a few nonEnglish studies were excluded due to incomplete data or unavailability of full text. (2) Our primary goal was to assess the diagnostic efficacy of ASCA in BD, and therefore we did not include all studies pertaining to ASCA in inflammatory bowel disease and iTB. (3) Restricted by the number of included studies and the isotypes of ASCA, we could not perform subgroup analysis disaggregated by different populations and diagnostic criteria. (4) Some studies with incomplete data were excluded after lack of response from the author. (5) There are some inherent statistical shortcomings using Meta-DiSc during the separate pooling of sensitivity and specificity, as the between-study variance is not included. More advanced methods are not implemented. ${ }^{32}$

\section{Conclusion}

Our study demonstrated the relationship between $\mathrm{ASCA} / S$. cerevisiae and gastrointestinal involvement in BD. Furthermore, ASCA may be detectable years before the diagnosis of some autoimmune diseases as they were retrospectively found in the preserved blood samples of soldiers who were affected by CD years later. ${ }^{33}$ However, detection of only ASCA may have a limited value for clinical diagnosis due to its moderate sensitivity and the presence in several other autoimmune diseases. In the future, further studies are needed to explore the role of ASCA and $S$. cerevisiae in BD.

Contributors $\mathrm{LC}$ and YL conceived the idea and design the analysis. LC, LL, CL and SY involved in acquisition of data. LC, LL and CL involved in statistical analysis and interpretation of data. LC drafted the manuscript. YL, LC and LL revised the manuscript. YL supervised the work. All authors read and approved the final manuscript.

Funding This research was supported by grants from the National Natural Science Foundation of China Grants $(81671618,81871302)$, CAMS Innovation Fund for Medical Sciences (CIFMS) (2017-I2M-3-001, 2017-I2M-B\&R-01).

Competing interests None declared.

Patient consent for publication Not required.

Ethics approval This article does not contain any studies with human participants performed by any of the authors.

Provenance and peer review Not commissioned; externally peer reviewed.

Data availability statement All data relevant to the study are included in the article or uploaded as supplementary information.

Supplemental material This content has been supplied by the author(s). It has not been vetted by BMJ Publishing Group Limited (BMJ) and may not have been peer-reviewed. Any opinions or recommendations discussed are solely those of the author(s) and are not endorsed by BMJ. BMJ disclaims all liability and responsibility arising from any reliance placed on the content. Where the content includes any translated material, BMJ does not warrant the accuracy and reliability of the translations (including but not limited to local regulations, clinical guidelines, terminology, drug names and drug dosages), and is not responsible for any error and/or omissions arising from translation and adaptation or otherwise.

Open access This is an open access article distributed in accordance with the Creative Commons Attribution Non Commercial (CC BY-NC 4.0) license, which permits others to distribute, remix, adapt, build upon this work non-commercially, and license their derivative works on different terms, provided the original work is properly cited, appropriate credit is given, any changes made indicated, and the use is non-commercial. See: http://creativecommons.org/licenses/by-nc/4.0/.

\section{ORCID iDs}

Linlin Cheng http://orcid.org/0000-0003-1924-3363

Yongzhe Li http://orcid.org/0000-0002-8267-0985

\section{REFERENCES}

1 Yazici H, Seyahi E, Hatemi G, et al. Behçet syndrome: a contemporary view. Nat Rev Rheumatol 2018;14:107-19.

2 Criteria for diagnosis of Behçet's disease. International study group for Behçet's disease. Lancet 1990;335:1078-80.

3 International Team for the Revision of the International Criteria for Behçet's Disease (ITR-ICBD). The International criteria for Behçet's disease (ICBD): a collaborative study of 27 countries on the sensitivity and specificity of the new criteria. J Eur Acad Dermatol Venereol 2014;28:338-47.

4 Alpsoy E, Donmez L, Bacanli A, et al. Review of the chronology of clinical manifestations in 60 patients with Behçet's disease. Dermatology 2003;207:354-6.

5 Sicard D, Legras J-L, Bread LJL. Bread, beer and wine: yeast domestication in the Saccharomyces sensu stricto complex. C R Biol 2011;334:229-36.

6 Aslan M, Kocazeybek B, Celik A, et al. Anti-saccharomyces cerevisiae (ASCA) antibody levels in a subgroup of patients with 
ulcerative colitis, Crohn's disease, Gi Behcet, and Gi tuberculosis: correlations with disease duration, activity, and extension. Int J Infect Dis 2010;14:e117-8.

7 Filik L, Biyikoglu I, Levent F, Ibrahim B. Differentiation of Behcet's disease from inflammatory bowel diseases: anti-Saccharomyces cerevisiae antibody and anti-neutrophilic cytoplasmic antibody. World J Gastroenterol 2008;14:7271.

8 Kocazeybek B, Aslan M, Erzin Y, et al. Clinical utility of perinuclear antineutrophil cytoplasmic antibodies and anti-Saccharomyces cerevisiae antibodies for discriminating specific intestinal inflammations. Int J Infect Dis 2010;14:e118.

9 Zhang S, Luo J, Li J, Shulan Z, Jing L, Ji L, et al. Retrospective evaluation of the clinical utility of serological biomarkers in Chinese patients with inflammatory bowel disease: 2-year clinical experience. Clin Chem Lab Med 2017:55:865-75.

10 Zhang S, Luo J, Wu Z, Shulan Z, Jing L, Ziyan W, et al. Antibodies against glycoprotein 2 display diagnostic advantages over ASCA in distinguishing CD from intestinal tuberculosis and intestinal Behçet's disease. Clin Trans/ Gastroenterol 2018;9:e133.

11 Lidar M, Langevitz P, Barzilai O, et al. Infectious serologies and autoantibodies in inflammatory bowel disease: insinuations at a true pathogenic role. Ann N Y Acad Sci 2009;1173:640-8.

12 McInnes MDF, Moher D, Thombs BD, et al. Preferred reporting items for a systematic review and meta-analysis of diagnostic test accuracy studies: the PRISMA-DTA statement. JAMA 2018;319:388-96

13 Stroup DF, Berlin JA, Morton SC, et al. Meta-analysis of observational studies in epidemiology: a proposal for reporting. meta-analysis of observational studies in epidemiology (moose) group. JAMA 2000;283:2008-12.

14 Liberati A, Altman DG, Tetzlaff J, et al. The PRISMA statement for reporting systematic reviews and meta-analyses of studies that evaluate healthcare interventions: explanation and elaboration. BMJ 2009;339:b2700.

15 Cheung MW-L, Ho RCM, Lim Y, et al. Conducting a meta-analysis: basics and good practices. Int J Rheum Dis 2012;15:129-35.

16 Krause I, Monselise Y, Milo G, et al. Anti-saccharomyces cerevisiae antibodies--a novel serologic marker for Behçet's disease. Clin Exp Rheumatol 2002;20:S21-4.

17 Monselise A, Weinberger A, Monselise Y, et al. Anti-Saccharomyces cerevisiae antibodies in Behçet's disease--a familial study. Clin Exp Rheumatol 2006;24:S87-90.

18 Prado LL, Augusto KL, Magalhães PF, et al. THU0219 Antisaccharomyces cerevisae antibodies (ASCA) in active behÇet's disease: A marker of intestinal involvement? Ann Rheum Dis 2013;71:229.2-229.

19 Oshitani N, Hato F, Jinno Y, et al. Igg subclasses of anti Saccharomyces cerevisiae antibody in inflammatory bowel disease. Eur J Clin Invest 2001;31:221-5.

20 Whiting PF, Rutjes AWS, Westwood ME, et al. QUADAS-2: a revised tool for the quality assessment of diagnostic accuracy studies. Ann Intern Med 2011;155:529-36.

21 Fresko I, Ugurlu S, Ozbakir F, et al. Anti-saccharomyces cerevisiae antibodies (ASCA) in Behçet's syndrome. Clin Exp Rheumatol 2005;23:S67-70.

22 Rinaldi M, Perricone R, Blank M, et al. Anti-saccharomyces cerevisiae autoantibodies in autoimmune diseases: from bread baking to autoimmunity. Clin Rev Allergy Immunol 2013;45:152-61.

23 Seibold F. ASCA: genetic marker, predictor of disease, or marker of a response to an environmental antigen? Gut 2005;54:1212-3.

24 Zouboulis CC, Büttner P, Tebbe B, et al. Anticardiolipin antibodies in Adamantiades-Behçet's disease. Br J Dermatol 1993;128:281-4.

25 Islam MA, Alam SS, Kundu S, et al. Prevalence of antiphospholipid antibodies in Behcet's disease: a systematic review and metaanalysis. PLoS One 2020;15:e0227836.

26 El-Lateef HMA. Behect disease in pediatrics; a solitary sign can be enough. Pediatr Rheumatol 2019;17.

27 Mizushima Y, Inaba G, Mimura Y, et al. Diagnostic criteria for Behçet's disease in 1987, and guideline for treating Behçet's disease, 1988: 391-3.

28 Chang HK, Lee SS, Bai HJ, et al. Validation of the classification criteria commonly used in Korea and a modified set of preliminary criteria for Behçet's disease: a multi-center study. Clin Exp Rheumatol 2004;22:S21-6.

29 Kramer M, Hasanreisoglu M, Weiss S, et al. Single-nucleotide polymorphisms in IL23R-IL12RB2 (rs1495965) are highly prevalent in patients with Behcet's uveitis and vary between populations. Ocul Immunol Inflamm 2019;27:766-73.

30 Fedrigo A, Skare TL, Bortoluzzi AL, et al. ASCA (Anti-saccharomyces cerevisiae antibody) in patients with scleroderma. J Clin Rheumatol 2019;25:24-7.
31 Holle JU, Hellmich B, Backes M, et al. Variations in performance characteristics of commercial enzyme immunoassay kits for detection of antineutrophil cytoplasmic antibodies: what is the optimal cut off? Ann Rheum Dis 2005;64:1773-9.

32 Junfeng W, Mariska L. Recommended software/packages for metaanalysis of diagnostic accuracy. J Lab Precis Med 2019;4.

33 Israeli E, Grotto I, Gilburd B, et al. Anti-saccharomyces cerevisiae and antineutrophil cytoplasmic antibodies as predictors of inflammatory bowel disease. Gut 2005;54:1232-6.

34 Vaiopoulos G, Lakatos PL, Papp M, et al. Serum anti-Saccharomyces cerevisiae antibodies in Greek patients with Behcet's disease. Yonsei Med J 2011:52:347-50.

$35 \mathrm{Choi} \mathrm{CH}$, Kim TI, Kim BC, et al. Anti-saccharomyces cerevisiae antibody in intestinal Behçet's disease patients: relation to clinical course. Dis Colon Rectum 2006;49:1849-59.

36 Rhee S-H, Kim Y-B, Lee E-S. Comparison of Behcet's disease and recurrent aphthous ulcer according to characteristics of gastrointestinal symptoms. J Korean Med Sci 2005;20:971-6.

$37 \mathrm{Kim}$ BG, Kim YS, Kim JS, et al. Diagnostic role of antiSaccharomyces cerevisiae mannan antibodies combined with antineutrophil cytoplasmic antibodies in patients with inflammatory bowel disease. Dis Colon Rectum 2002:45:1062-9.

38 de Vries M, van der Horst-Bruinsma I, van Hoogstraten I, et al. pANCA, ASCA, and ompC antibodies in patients with ankylosing spondylitis without inflammatory bowel disease. J Rheumatol 2010;37:2340-4.

39 Riente L, Chimenti D, Pratesi F, et al. Antibodies to tissue transglutaminase and Saccharomyces cerevisiae in ankylosing spondylitis and psoriatic arthritis. J Rheumatol 2004;31:920-4.

40 Mundwiler ML, Mei L, Landers CJ, et al. Inflammatory bowel disease serologies in ankylosing spondylitis patients: a pilot study. Arthritis Res Ther 2009;11:R177.

41 Wallis D, Asaduzzaman A, Weisman M, Dinny W, Arundip A, Michael W, et al. Elevated serum anti-flagellin antibodies implicate subclinical bowel inflammation in ankylosing spondylitis: an observational study. Arthritis Res Ther 2013;15:R166.

42 Krause I, Blank M, Cervera R, et al. Cross-reactive epitopes on beta2-glycoprotein-I and Saccharomyces cerevisiae in patients with the antiphospholipid syndrome. Ann N Y Acad Sci 2007:1108:481-8

43 Gonzalez T, Malagon C, Guarnizo P, et al. Autoantibodies and gastrointestinal symptoms in Colombian children with juvenile idiopathic arthritis. Curr Rheumatol Rev 2018;14:163-71.

44 Muratori P, Muratori L, Guidi M, et al. Anti-saccharomyces cerevisiae antibodies (ASCA) and autoimmune liver diseases. Clin Exp Immunol 2003:132:473-6.

45 Czaja AJ, Shums Z, Donaldson PT, et al. Frequency and significance of antibodies to Saccharomyces cerevisiae in autoimmune hepatitis. Dig Dis Sci 2004;49:611-8.

46 Papp M, Norman GL, Vitalis Z, et al. Presence of anti-microbia antibodies in liver cirrhosis--a tell-tale sign of compromised immunity? PLoS One 2010;5:e12957.

47 Sakly W, Jeddi M, Ghedira I. Anti-saccharomyces cerevisiae antibodies in primary biliary cirrhosis. Dig Dis Sci 2008;53:1983-7.

48 Main J, McKenzie H, Yeaman GR, et al. Antibody to Saccharomyces cerevisiae (bakers' yeast) in Crohn's disease. BMJ 1988;297:1105-6.

49 Shor DB-A, Orbach H, Boaz M, et al. Gastrointestinal-associated autoantibodies in different autoimmune diseases. Am J Clin Exp Immunol 2012;1:49-55.

50 Yazıcı D, Aydın SZ, Yavuz D, et al. Anti-saccaromyces cerevisiae antibodies (ASCA) are elevated in autoimmune thyroid disease ASCA in autoimmune thyroid disease. Endocrine 2010;38:194-8.

51 Mankaï A, Thabet Y, Manoubi W, et al. Anti-saccharomyces cerevisiae antibodies are elevated in Graves' disease but not in Hashimoto's thyroiditis. Endocr Res 2013;38:98-104.

52 Dai H, Li Z, Zhang Y, et al. Elevated levels of serum IgA against Saccharomyces cerevisiae mannan in patients with rheumatoid arthritis. Cell Mol Immunol 2009;6:361-6.

53 Dai H, Li Z, Zhang Y, et al. Elevated levels of serum antibodies against Saccharomyces cerevisiae mannan in patients with systemic lupus erythematosus. Lupus 2009;18:1087-90.

54 Mankaï A, Sakly W, Thabet Y, et al. Anti-saccharomyces cerevisiae antibodies in patients with systemic lupus erythematosus. Rheumatol Int 2013;33:665-9.

55 Sakly W, Mankaï A, Sakly N, et al. Anti-saccharomyces cerevisiae antibodies are frequent in type 1 diabetes. Endocr Pathol 2010;21:108-14.

56 Alunno A, Bistoni O, Carubbi F, et al. Prevalence and significance of anti-saccharomyces cerevisiae antibodies in primary Sjögren's syndrome. Clin Exp Rheumatol 2018;36:73-9. 